\title{
COVID-19: When recovery does not mean a return to health
}

\author{
SADJ July 2020, Vol. 75 No. 6 p286
}

NH Wood

Managing Editor of the SADJ

The number of COVID-19 cases in South Africa is steadily increasing. The reports and daily updates we receive from the National Department of Health and from the National Institute for Communicable Diseases (NICD) reflect the daily numbers of new cases, of deaths, and the number of recoveries.

It is the latter category that is more ominous than the label implies. What does it mean when "recoveries" are reported? Those cases that have not demised as a direct result of SARS-CoV-2 infection, and whom have surpassed the number of days to be considered free from SARS-CoV-2 infection, are listed as having recovered from COVID-19.

What the recovery figure does not tell us, is that there are several multi-system effects that manifest long after the virus has cleared from the body. The pulmonary effects that range from mild to severe residual lung abnormalities are the first to receive attention in the literature. Some studies are focussed on the deleterious cardiac effects that result after persistent inflammation. ${ }^{1}$ Still more are reporting on chronic fatigue.

The final form of any post-COVID-19 chronic conditions is yet to be revealed, and the rehabilitation of these patients may take years to achieve. The ongoing impact of these "recoveries" should therefore not be underestimated. The long-term influence and strain on the healthcare system, on the economy and the education system to name only a few is undetermined as yet.

The situation is compounded by the recent $\mathrm{WHO}$ report $^{2}$ in which the lack of studies into antibody development and into antibody-mediated immunity against SARS-CoV-2 is highlighted. The risk for reinfection, and subsequent further transmission and spread is deeply concerning within the relative data vacuum.

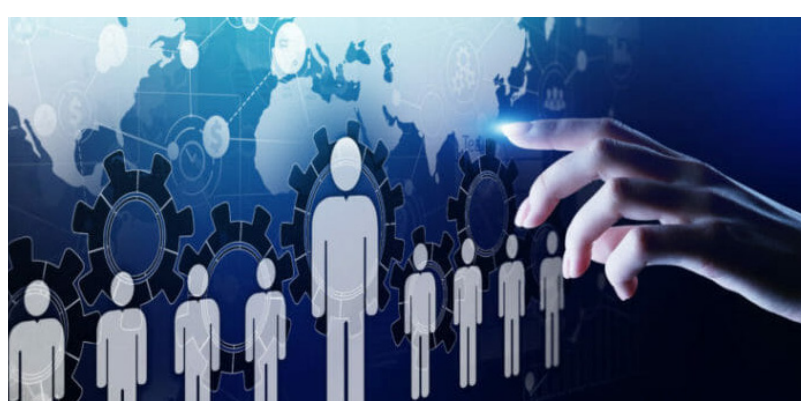

Neil H Wood: Managing editor. Email: neil.wood@smu.ac.za

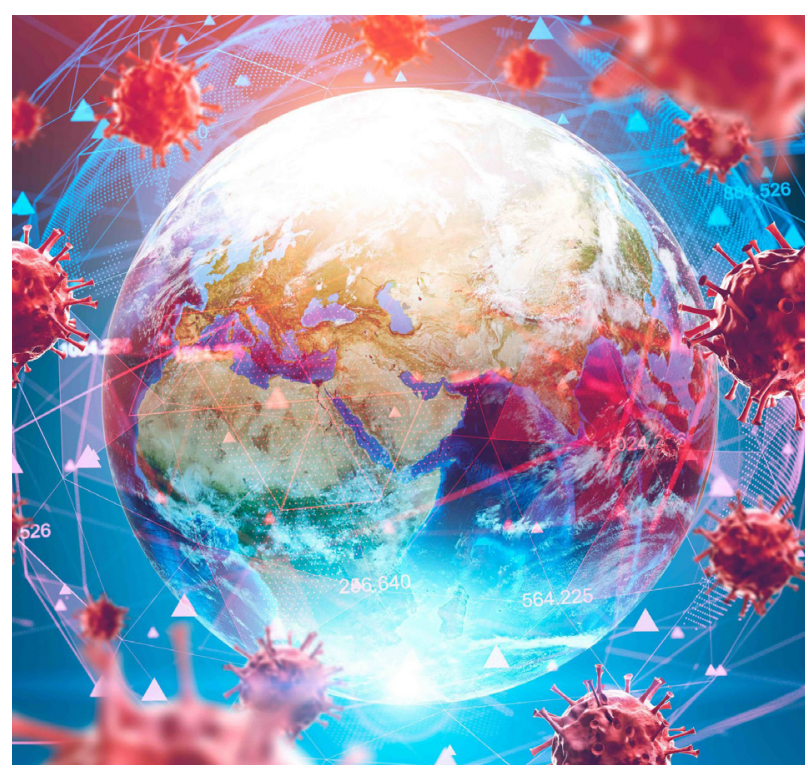

The lag in COVID-19 testing, and number of tests currently performed must also be taken into account.

We are all responsible for the lives of others during this pandemic. Please be reminded to remain safe: don't let your guard down, maintain distancing, look out for potential super-spreading events, and be vigilant with regards to infection control against the spread of SARSCoV-2. Someone else's life depends on your behaviour.

I thank Dr NP Metsing who briefly shares with us her experience having dealt with COVID-19. At this point in time we all know someone who has been infected or directly affected by this virus. Some of us have already lost loved ones, colleagues and friends.

We extend our well-wishes to those who are currently fighting this infection, and to those who have loved ones currently infected. Thank you also to all our contributors to this issue of the South African Dental Journal.

\section{Reference}

1. Madjid M, Safavi-Naeini P, Solomon SD, Vardeny O. Potential Effects of Coronaviruses on the Cardiovascular System: A Review. JAMA Cardiology. 2020; DOI: 10.1001/ jamacardio.2020.1286

2. World Health Organisation. "Immunity passports" in the context of COVID-19 https://www.who.int/news-room/commentaries/detail/immunity-passports-in-the-context-of-covid-19? gclid=CjwKCAjw9vn4BRBaEiwAhOmuDIEBkyy4RTvfumy4mNa4v039DzT-xmp5h_KkijXC4zcVA4Ktbef7uxoCOJEQAvD_ BwE. 